\title{
\begin{tabular}{l|l} 
Mitraries & DSpace@MIT
\end{tabular}
}

\author{
MIT Open Access Articles
}

\section{Hysteresis-Based Switching Design for Stabilization of Switched Linear Neutral Systems}

The MIT Faculty has made this article openly available. Please share how this access benefits you. Your story matters.

Citation: Li, Tai-Fang, Jun Fu, and Ben Niu. “Hysteresis-Based Switching Design for Stabilization of Switched Linear Neutral Systems." Circuits, Systems, and Signal Processing 36, no. 1 (March 18, 2016): 359-373.

As Published: http://dx.doi.org/10.1007/s00034-016-0294-7

Publisher: Springer US

Persistent URL: http://hdl.handle.net/1721.1/106402

Version: Author's final manuscript: final author's manuscript post peer review, without publisher's formatting or copy editing

Terms of Use: Article is made available in accordance with the publisher's policy and may be subject to US copyright law. Please refer to the publisher's site for terms of use. 


\title{
Hysteresis-Based Switching Design for Stabilization of Switched Linear Neutral Systems
}

\author{
Tai-Fang Li · Jun Fu • Ben Niu
}

Received: date / Accepted: date

\begin{abstract}
The paper studies the problem of asymptotically stabilizing a class of switched linear neutral systems, where none of the individual subsystems is stabilizable. A co-design of a set of candidate controllers and a hysteresisbased switching strategy is developed to achieve asymptotic stability of the closed-loop switched neutral systems. Finally, a numerical example is given to illustrate the effectiveness of the proposed method.
\end{abstract}

Keywords Switched neutral systems · hysteresis-based switching · multiple Lyapunov-Krasovskii functionals $\cdot$ stabilization

\section{Introduction}

Switched systems have attracted lots of attention in the last two decades [1, $16,21,34]$. A switched system is a dynamical system that consists of a finite number of subsystems and a logical rule that orchestrates switching between these subsystems [17]. There have been lots of works concerned with switched systems, see, for example, $[4,5,18,22,23,26,30,37,40,41]$. Moreover, switching control strategies also play important roles in control systems. [27] uses multiple controllers switching strategy to stabilize a class of linear neutral systems

Tai-Fang Li

College of Engineering, Bohai University, Jinzhou, China, 121013

E-mail: xiaofang0412@163.com

Jun Fu

State Key Laboratory of Synthetical Automation for Process Industries, Northeastern University, China, 110189, and Department of Mechanical Engineering, Massachusetts Institute of Technology (MIT), Cambridge, MA, USA, 02139

E-mail: junfu@mit.edu

Ben Niu

College of Mathematics and Physics, Bohai University, Jinzhou, China, 121013

E-mail: niuben20062007@126.com 
and first proposes the definition of the switched neutral system. The switched neutral system, i.e., a switched system whose subsystems are a family of neutral systems (see $[3,6-9,11,24,42]$ for the details of neutral systems), has drawn considerable attention due to its importance from both theoretical $[10,19,28$, $32]$ and practical points of view $[25,27]$. Recent years have seen lots of research activities focusing on stability analysis and stabilization of switched neutral systems, where designing switching signals using different methods plays an important role. Among these technical methods, time-dependent average dwell time methods $[12,13,29,31,35]$ and state-dependent multiple Lyapunov function approaches $[14,33,38]$ are often adopted. Since it is often difficult to $e x$ plicitly determine the switching instants in practice, state-dependent switching strategies have a wider application range than time-dependent switching ones. Therefore, this paper will focus on the design of state-dependent switching strategy to stabilize switched neutral systems.

For the considered problem, the authors of $[14,33,38]$ study stabilization problem of switched linear neutral systems using state-dependent switching strategies. However, these designed switching signals in $[14,33,38]$ cannot avoid sliding modes or chattering behaviors, i.e. infinitely fast switchings occurring on switching surfaces, which are often undesirable because in practice they may cause excessive equipment wear. [20] presents a hysteresis switching strategy in control design to avoid sliding modes and chattering behaviors. [15] applies a hysteresis-based switching strategy to stabilize switched neutral systems. However, single Lyapunov-Krasovskii functional method adopted in [15] requires stability of the convex combination system, which brings a certain degree of conservativeness. As we all know that the multiple Lyapunov function method is a less conservative stability analysis tool for switched systems $[2,16$, 17,39]. Thus, questions naturally arise: is it possible to achieve stabilization of switched neutral systems using hysteresis-based switching strategy in the framework of the multiple Lyapunov-Krasovskii functional approach? If possible, under what conditions can we achieve this goal and how? To the best of our knowledge, there have not been results in the literature which provide answers to these questions. This motivates the present paper.

From the above motivation, we first propose a hysteresis-based switching strategy by generalizing the well-known min-switching strategy, then introduce a multiple generalized Lyapunov-Krasovskii functional method, which allows some increase of connecting adjacent Lyapunov-Krasovskii functionals at switching points, last derive a delay-dependent sufficient condition to guarantee asymptotic stability of the system.

The paper is organized as follows. Section 2 gives problem statement and required preliminaries. Section 3 presents the main result. Section 4 illustrates the effectiveness of the proposed method with a numerical example. Section 5 concludes this paper.

Notation Throughout this paper, $\mathbb{R}^{n}$ is the $n$-dimensional Euclidean space. $\mathbb{N}$ is the set of nonnegative integers. For a square matrix $P, P>0$ means that $P$ is positive definite; $P^{T}$ and $P^{-1}$ denote the transpose and the inverse of the matrix $P$, respectively. $\|\cdot\|$ denotes the Euclidean vector norm. $*$ denotes the 
elements below the main diagonal of a symmetric matrix. $\operatorname{diag}\{\cdots\}$ denotes a block-diagonal matrix.

\section{Problem statement and preliminaries}

Consider the following switched neutral system

$$
\left\{\begin{array}{l}
\dot{x}(t)-C_{\sigma} \dot{x}(t-h)=A_{\sigma} x(t)+B_{\sigma} x(t-\tau)+D_{\sigma} u(t), \quad t>0 \\
x_{0}=\varphi(\theta), \quad \theta \in[-\max \{h, \tau\}, 0]
\end{array}\right.
$$

where $x(t) \in \mathbb{R}^{n}$ is the system state, $u(t) \in \mathbb{R}^{m}$ is the control input, $h$ and $\tau$ are time delays, $\varphi(\theta)$ is a continuously differentiable initial function on $[-\max \{h, \tau\}, 0] . \sigma:[0, \infty) \rightarrow \mathcal{M}=\{1,2, \cdots, m\}$ is a piecewise constant function called switching signal. Corresponding to $\sigma$, there exists a switching sequence $\left\{x_{t_{0}}:\left(i_{0}, t_{0}\right), \cdots,\left(i_{k}, t_{k}\right), \cdots \mid i_{k} \in \mathcal{M}, k \in \mathbb{N}\right\}$, which means that the subsystem $i_{k}$ is active when $t \in\left[t_{k}, t_{k+1}\right) . A_{i}, B_{i}, C_{i}$ and $D_{i}, i \in \mathcal{M}$, are known real matrices which define the subsystem $i$ and $\left\|C_{i}\right\|<1$. Without loss of generality, we assume that $t_{0}=0$ and the state trajectory $x(\cdot)$ of the system (1) is continuous everywhere.

The feedback controller of the system (1) is designed as

$$
u(t)=u_{\sigma}(t)=K_{1 \sigma} x(t)+K_{2 \sigma} x(t-\tau),
$$

where $K_{1 i}$ and $K_{2 i}$ are controller gains of the subsystem $i$.

From (2), the closed-loop system (1) can be written as

$$
\dot{x}(t)-C_{\sigma} \dot{x}(t-h)=\left(A_{\sigma}+D_{\sigma} K_{1 \sigma}\right) x(t)+\left(B_{\sigma}+D_{\sigma} K_{2 \sigma}\right) x(t-\tau) .
$$

We now introduce two useful lemmas for our main result.

Lemma 1[42] For any constant matrix $M>0$, scalars $r_{1}$ and $r_{2}$ satisfying $r_{1}<r_{2}$, and a vector function $\omega:\left[r_{1}, r_{2}\right] \rightarrow \mathbb{R}^{n}$ such that the integrations concerned are well defined, then

$$
-\left(r_{2}-r_{1}\right) \int_{r_{1}}^{r_{2}} \omega^{T}(s) M \omega(s) d s \leq-\int_{r_{1}}^{r_{2}} \omega^{T}(s) d s M \int_{r_{1}}^{r_{2}} \omega(s) d s .
$$

Lemma 2[16] For any symmetric matrices $T_{0}$ and $T_{1}$ with $x^{T} T_{1} x \geq 0$ subject to $x \neq 0$, if there exist a scalar $\beta \geq 0$, such that $T_{0}-\beta T_{1}>0$, then $x^{T} T_{0} x>0$.

\section{Main Result}

It is well known that the min-switching strategy is a classical switching strategy in the switched system field. [16] proposes the min-switching strategy for switched linear systems by partitioning the state space $\mathbb{R}^{n}$ into $m$ regions:

$$
\begin{gathered}
\Omega_{i}=\left\{x \in \mathbb{R}^{n} \mid x^{T}\left(P_{i}-P_{j}\right) x \leq 0, \forall i, j \in \mathcal{M}\right\}, \\
\Omega_{i j}=\left\{x \in \mathbb{R}^{n} \mid x^{T}\left(P_{i}-P_{j}\right) x=0, \forall i, j \in \mathcal{M}\right\},
\end{gathered}
$$


where $P_{i}>0$ and $P_{j}>0$. Switchings only occur on switching surfaces $x^{T} P_{i} x=$ $x^{T} P_{j} x$ according to the switching law

$$
\sigma:=\operatorname{argmin}\left\{V_{i}(x): \forall i \in \mathcal{M}\right\},
$$

where $V_{i}(x)=x^{T} P_{i} x$. From (5) and (7), we know that the Lyapunov function $V_{\sigma}(x)$ is continuous at all switching points. Furthermore, although the stability property can be retained by adopting the switching strategy (7), sliding modes and chattering behaviors cannot be avoided. In order to relax the continuous constraint on $V_{\sigma}(x)$ and avoid sliding modes, we redefine $m$ regions:

$$
\begin{aligned}
& \Omega_{i}=\left\{x \in \mathbb{R}^{n} \mid x^{T}\left(P_{i}-P_{j}+N_{i j}\right) x \leq 0, \forall i, j \in \mathcal{M}, i \neq j\right\}, \\
& \Omega_{i j}=\left\{x \in \mathbb{R}^{n} \mid x^{T}\left(P_{i}-P_{j}+N_{i j}\right) x=0, \forall i, j \in \mathcal{M}, i \neq j\right\},
\end{aligned}
$$

where $P_{i}>0, P_{j}>0, N_{i j}$ are symmetric matrices satisfying $N_{i j}+N_{j k} \leq N_{i k}$ and $N_{i i}=0$ for $\forall i, j, k \in \mathcal{M}$. It is obvious that $\Omega_{i j}$ is the boundary of $\Omega_{i}$ and

$$
\bigcup_{i=1}^{m} \Omega_{i}=\mathbb{R}^{n}
$$

In fact, if (10) does not hold, there exists $x \in \mathbb{R}^{n}$ satisfying $x \notin \Omega_{i}$, then we have an integer $q$ and a sequence $i_{1}, i_{2}, \cdots, i_{q}, i_{k} \neq i_{k+1}, k=1, \cdots, q, i_{q+1}=i_{1}$ such that

$$
x^{T}\left(P_{i_{k}}-P_{i_{k+1}}+N_{i_{k} i_{k+1}}\right) x>0, \quad \forall i_{k} \in \mathcal{M} .
$$

Taking the sum over $k$ and noticing $N_{i j}+N_{j k} \leq N_{i k}$, and $N_{i i}=0$, we have

$$
\sum_{k=1}^{q} x^{T}\left(P_{i_{k}}-P_{i_{k+1}}+N_{i_{k} i_{k+1}}\right) x=\sum_{k=1}^{q} x^{T} N_{i_{k} i_{k+1}} x \leq 0,
$$

which contradicts (11). Hence, (10) holds. We thus design a hysteresis-based switching strategy based on (8):

$$
\left\{\begin{array}{l}
t=0, \sigma(0)= \begin{cases}i, & \text { if } x(0) \in \Omega_{i} / \Omega_{i} \bigcap\left(\Omega_{j} \mid j \in \mathcal{M}, j \neq i\right) \\
\operatorname{minarg}\left\{\Omega_{i}, \Omega_{j}\right\}, & \text { if } x(0) \in \Omega_{i} \bigcap\left(\Omega_{j} \mid j \in \mathcal{M}, j \neq i\right)\end{cases} \\
t>0, \sigma(t)= \begin{cases}i, & \text { if } x(t) \in \Omega_{i} \text { and } \sigma\left(t^{-}\right)=i \\
\operatorname{minarg}\left\{\Omega_{j} \mid j \in \mathcal{M}\right\}, & \text { if } x(t) \in \Omega_{i j} \text { and } \sigma\left(t^{-}\right)=i .\end{cases}
\end{array}\right.
$$

The switching law (13) can be described as follows. Let $\sigma(0)=i$ if $x(0) \in$ $\Omega_{i}$ and $\sigma(0)=\operatorname{minarg}\left\{\Omega_{i}, \Omega_{j}\right\}$ if $x(0)$ belongs to the overlapping area of some partition regions. For $t>0$, if $\sigma\left(t^{-}\right)=i$ and $x(t) \in \Omega_{i}$, the state trajectory remains in $\Omega_{i}$ until it hits the boundary $\Omega_{i j}$. This means that switching only takes place on the boundary $\Omega_{i j}$. If $\sigma\left(t^{-}\right)=i$ and $x(t) \in \Omega_{i j}$, then $x(t)$ enters $\Omega_{j_{\min }}, j_{\min }=\operatorname{minarg}\left\{\Omega_{j}\right\}$. In fact, $x(t) \in \Omega_{i j}$ means that $x^{T}\left(P_{i}-P_{j_{\min }}+N_{i j_{\min }}\right) x=0$ and $x^{T}\left(P_{i}-P_{j}+N_{i j}\right) x=0$ for $j \neq j_{\min }$. Thus, we have $x^{T} P_{j_{\min }} x=x^{T}\left(P_{i}+N_{i j_{\min }}\right) x$. This in turn gives

$$
\begin{aligned}
x^{T}\left(P_{j_{\min }}-P_{j}+N_{j_{\min } j}\right) x & =x^{T}\left(P_{i}+N_{i j_{\min }}-P_{j}+N_{j_{\min } j}\right) x \\
& \leq x^{T}\left(P_{i}-P_{j}+N_{i j}\right) x=0 .
\end{aligned}
$$


Remark 1 In order to design a hysteresis-based switching strategy avoiding sliding modes and chattering behaviors, we introduce $x^{T} N_{i j} x$ when partitioning the state space $\mathbb{R}^{n}$ and thus obtain $m$ overlapping regions, as shown in (8). However, the condition $x^{T}\left(N_{i j}+N_{j k}\right) x \leq x^{T} N_{i k} x$ is necessary to guarantee that the partition regions cover the entire state space $\mathbb{R}^{n}$.

Remark 2 From (9) and (13), $x^{T} N_{i j} x$ determines the monotony of $V_{\sigma}$ at switching points. Especially for $x^{T} N_{i j} x=0, x \neq 0$, the connecting adjacent Lyapunov functions are continuous at switching points, under which the switching strategy (13) degenerates into the min-switching strategy (7) exactly. The influencing behaviors are illustrated in Fig.1.

We now analyze stability of the closed-loop system (3). The following theorem gives the main result.

Theorem 1 Consider the system (3). If there exist scalars $\left.\alpha_{i j}\langle 0, \tau\rangle 0, h\right\rangle$ 0 , matrices $\bar{P}_{i}>0, \bar{P}_{j}>0, \forall j \in \mathcal{M}, j \neq i, \bar{R}_{1}>0, \bar{R}_{2}>0, \bar{Q}_{1}>0, \bar{Q}_{2}>$ $0, Z_{1 i}, Z_{2 i}, \bar{M}>0$, and $\bar{N}_{i j}$ such that

$$
\begin{aligned}
& {\left[\begin{array}{cccccc}
\Psi_{i j}^{11} & \bar{M} A_{i}^{T}+Z_{1 i}^{T} D_{i}^{T}+\bar{P}_{i}-\bar{M} & B_{i} \bar{M}+D_{i} Z_{2 i} & \frac{1}{h} \bar{R}_{2} & C_{i} \bar{M} & 0 \\
* & \bar{R}_{1}+h \bar{R}_{2}-2 \bar{M} & B_{i} \bar{M}+D_{i} Z_{2 i} & 0 & C_{i} \bar{M} & 0 \\
* & * & -\bar{Q}_{1} & 0 & 0 & 0 \\
* & * & * & -\frac{1}{h} \bar{R}_{2} & 0 & 0 \\
* & * & * & * & -\bar{R}_{1} & 0 \\
* & * & * & * & * & -\frac{1}{\tau} \bar{Q}_{2}
\end{array}\right]<0,} \\
& {\left[\begin{array}{cccc}
\Pi_{i j}^{11} & \Pi_{i j}^{12} & \bar{M} A_{i}^{T}+Z_{1 i}^{T} D_{i}^{T}+C_{i} \bar{M} & \bar{M} A_{i}^{T}+Z_{1 i}^{T} D_{i}^{T}-\bar{M}+\bar{N}_{i j}^{T} \\
* & \Pi_{i j}^{22} & \bar{M} B_{i}^{T}+Z_{2 i}^{T} D_{i}^{T}+C_{i} \bar{M} & \bar{M} B_{i}^{T}+Z_{2 i}^{T} D_{i}^{T}-\bar{M} \\
* & * & C_{i} \bar{M}+\bar{M} C_{i}^{T} & -\bar{M}+\bar{M} C_{i}^{T} \\
* & * & * & -2 \bar{M}
\end{array}\right]<0,} \\
& \bar{N}_{i j}+\bar{N}_{j k} \leq \bar{N}_{i k}, \forall i, j, k \in \mathcal{M},
\end{aligned}
$$

where

$$
\begin{aligned}
\Psi_{i j}^{11}= & \bar{Q}_{1}+\tau \bar{Q}_{2}-\frac{1}{h} \bar{R}_{2}+A_{i} \bar{M}+D_{i} Z_{1 i}+\bar{M} A_{i}^{T}+Z_{1 i}^{T} D_{i}^{T} \\
& +\sum_{j=1}^{m} \alpha_{i j}\left(\bar{P}_{i}-\bar{P}_{j}+\bar{N}_{i j}\right) \\
\Pi_{i j}^{11}= & A_{i} M+D_{i} Z_{1 i}+\bar{M} A_{i}^{T}+Z_{1 i}^{T} D_{i}^{T} \\
\Pi_{i j}^{12}= & \bar{M} A_{i}^{T}+Z_{1 i}^{T} D_{i}^{T}+B_{i} \bar{M}+D_{i} Z_{2 i} \\
\Pi_{i j}^{22}= & B_{i} \bar{M}+D_{i} Z_{2 i}+\bar{M} B_{i}^{T}+Z_{2 i}^{T} D_{i}^{T}
\end{aligned}
$$

then the system (3) is asymptotically stable under the switching law (13) where $P_{i}=\bar{M}^{-1} \bar{P}_{i} \bar{M}^{-1}, P_{j}=\bar{M}^{-1} \bar{P}_{j} \bar{M}^{-1}$, and $N_{i j}=\bar{M}^{-1} \bar{N}_{i j} \bar{M}^{-1}$, and the controller gains can be obtained by $K_{1 i}=Z_{1 i} \bar{M}^{-1}$ and $K_{2 i}=Z_{2 i} \bar{M}^{-1}$.

Proof Construct a Lyapunov-Krasovskii functional for the subsystem $i$ of the system (3) as follows

$$
\begin{aligned}
V_{i}(t)= & x^{T}(t) P_{i} x(t)+\int_{t-\tau}^{t} x^{T}(s) Q_{1} x(s) d s+\int_{-\tau}^{0} \int_{t+\theta}^{t} x^{T}(s) Q_{2} x(s) d s d \theta \\
& +\int_{t-h}^{t} \dot{x}^{T}(s) R_{1} \dot{x}(s) d s+\int_{-h}^{0} \int_{t+\theta}^{t} \dot{x}^{T}(s) R_{2} \dot{x}(s) d s d \theta
\end{aligned}
$$




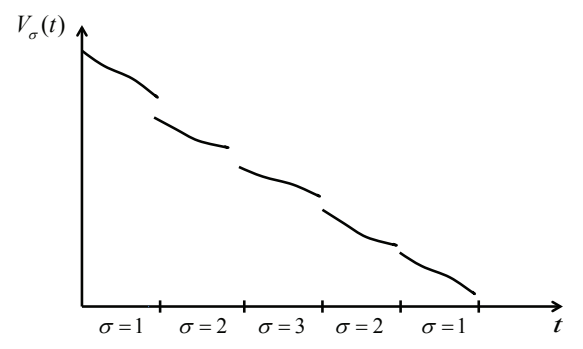

(a)

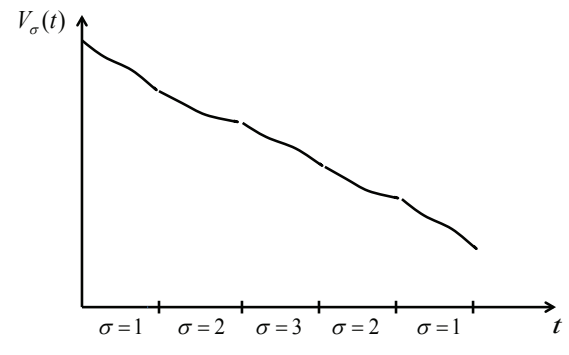

(b)

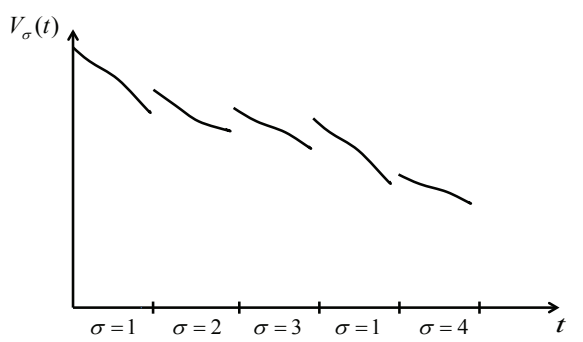

(c)

Fig. 1 Multiple Lyapunov functions (a) $x^{T} N_{i j} x<0$ (b) $x^{T} N_{i j} x=0$ (c) $x^{T} N_{i j} x>0$ 
where $P_{i}>0, Q_{1}>0, Q_{2}>0, R_{1}>0$, and $R_{2}>0$. When the subsystem $i$ is active on the interval $\left[t_{k}, t_{k+1}\right)$, differentiating (17) along solutions of the system (3) gives

$$
\begin{aligned}
\dot{V}_{i}(t)= & 2 \dot{x}^{T}(t) P_{i} x(t)+x^{T}(t) Q_{1} x(t)-x^{T}(t-\tau) Q_{1} x(t-\tau) \\
& +\dot{x}^{T}(t) R_{1} \dot{x}(t)-\dot{x}^{T}(t-h) R_{1} \dot{x}(t-h)+\tau x^{T}(t) Q_{2} x(t) \\
& -\int_{t-\tau}^{t} x^{T}(s) Q_{2} x(s) d s+h \dot{x}^{T}(t) R_{2} \dot{x}(t)-\int_{t-h}^{t} \dot{x}^{T}(s) R_{2} \dot{x}(s) d s .
\end{aligned}
$$

From Lemma 1, we have

$$
\begin{aligned}
&-\int_{t-\tau}^{t} x^{T}(s) Q_{2} x(s) d s \leq-\frac{1}{\tau} \int_{t-\tau}^{t} x^{T}(s) d s Q_{2} \int_{t-\tau}^{t} x(s) d s, \\
&-\int_{t-h}^{t} \dot{x}^{T}(s) R_{2} \dot{x}(s) d s \leq-\frac{1}{h} \int_{t-h}^{t} \dot{x}^{T}(s) d s R_{2} \int_{t-h}^{t} \dot{x}(s) d s \\
&=-\frac{1}{h}\left(x^{T}(t)-x^{T}(t-h)\right) R_{2}(x(t)-x(t-h)) .
\end{aligned}
$$

Moreover, from the system (3), for an arbitrary matrix $M$, we have

$$
\begin{aligned}
2\left(x^{T}(t) \dot{x}^{T}(t)\right) M & \left(\left(A_{i}+D_{i} K_{1 i}\right) x(t)\right. \\
& \left.+\left(B_{i}+D_{i} K_{2 i}\right) x(t-\tau)+C_{i} \dot{x}(t-h)-\dot{x}(t)\right)=0 .
\end{aligned}
$$

Combining (19) and (20) and inserting (21) into (18), we have

$$
\dot{V}_{i}(t) \leq \xi^{T}(t) \Phi_{i} \xi(t)
$$

where

$$
\begin{gathered}
\xi^{T}(t)=\left(\begin{array}{llllll}
x^{T}(t) & \dot{x}^{T}(t) & x^{T}(t-\tau) & x^{T}(t-h) & \dot{x}^{T}(t-h) & \int_{t-\tau}^{t} x^{T}(s) d s
\end{array}\right) \\
\Phi_{i}=\left[\begin{array}{cccccc}
\Phi_{i}^{11} & \left(A_{i}+D_{i} K_{1 i}\right)^{T} M+P_{i}-M & M\left(B_{i}+D_{i} K_{2 i}\right) & \frac{1}{h} R_{2} & M C_{i} & 0 \\
* & R_{1}+h R_{2}-2 M & M\left(B_{i}+D_{i} K_{2 i}\right) & 0 & M C_{i} & 0 \\
* & * & -Q_{1} & 0 & 0 & 0 \\
* & * & * & -\frac{1}{h} R_{2} & 0 & 0 \\
* & * & * & * & -R_{1} & 0 \\
* & * & * & * & * & -\frac{1}{\tau} Q_{2}
\end{array}\right], \\
\Phi_{i}^{11}=Q_{1}+\tau Q_{2}-\frac{1}{h} R_{2}+M\left(A_{i}+D_{i} K_{1 i}\right)+\left(A_{i}+D_{i} K_{1 i}\right)^{T} M
\end{gathered}
$$

When the subsystem $i$ is active on the interval $\left[t_{k}, t_{k+1}\right), \sum_{j=1}^{m} \alpha_{i j} x^{T}(t)\left(P_{i}-\right.$ $\left.P_{j}+N_{i j}\right) x(t) \geq 0$ follows from the switching law (13) and the condition $\alpha_{i j}<0$. Then, Lemma 2 allows us to replace the condition $\Phi_{i}<0$ by

$$
\left[\begin{array}{cccccc}
\bar{\Phi}_{i}^{11}\left(A_{i}+D_{i} K_{1 i}\right)^{T} M+P_{i}-M & M\left(B_{i}+D_{i} K_{2 i}\right) & \frac{1}{h} R_{2} & M C_{i} & 0 \\
* & R_{1}+h R_{2}-2 M & M\left(B_{i}+D_{i} K_{2 i}\right) & 0 & M C_{i} & 0 \\
* & * & -Q_{1} & 0 & 0 & 0 \\
* & * & * & -\frac{1}{h} R_{2} & 0 & 0 \\
* & * & * & * & -R_{1} & 0 \\
* & * & * & * & * & -\frac{1}{\tau} Q_{2}
\end{array}\right]<0,
$$


where

$\bar{\Phi}_{i j}^{11}=Q_{1}+\tau Q_{2}-\frac{1}{h} R_{2}+M\left(A_{i}+D_{i} K_{1 i}\right)+\left(A_{i}+D_{i} K_{1 i}\right)^{T} M+\sum_{j=1}^{m} \alpha_{i j}\left(P_{i}-P_{j}+N_{i j}\right)$.

Hence, the inequality $(23)$ implies $\dot{V}_{i}(t)<0$, which means $V_{i}(t)$ is strictly decreasing on the interval $\left[t_{k}, t_{k+1}\right)$. Obviously, the condition (23) is a nonlinear matrix inequality. Multiplying both sides of the inequality (23) by $\operatorname{diag}\left\{M^{-1}, \cdots, M^{-1}\right\}$, and letting $\bar{M}=M^{-1}, \bar{Q}_{1}=\bar{M} Q_{1} \bar{M}, \bar{Q}_{2}=\bar{M} Q_{2} \bar{M}$, $\bar{P}_{i}=\bar{M} P_{i} \bar{M}, \bar{P}_{j}=\bar{M} P_{j} \bar{M}, \bar{R}_{1}=\bar{M} R_{1} \bar{M}, \bar{R}_{2}=\bar{M} R_{2} \bar{M}, Z_{1 i}=K_{1 i} \bar{M}$, $Z_{2 i}=K_{2 i} \bar{M}$, and $\bar{N}_{i j}=\bar{M} N_{i j} \bar{M}$, we obtain the equivalent condition (14) which can be easily solved by using MATLAB LMI Toolbox.

Moreover, from the system (3), we have

$$
\begin{aligned}
& 2\left(x^{T}(t) \quad x^{T}(t-\tau) \quad \dot{x}^{T}(t-h) \quad \dot{x}^{T}(t)\right) M\left(\left(A_{i}+D_{i} K_{1 i}\right) x(t)\right. \\
& \left.+\left(B_{i}+D_{i} K_{2 i}\right) x(t-\tau)+C_{i} \dot{x}(t-h)-\dot{x}(t)\right)=0 .
\end{aligned}
$$

Let $V_{i j}(t)=x^{T}(t) N_{i j} x(t)$. When the subsystem $i$ is active on the interval $\left[t_{k}, t_{k+1}\right)$, differentiating $V_{i j}(t)$ along solutions of the system (3) and adding (24) gives

$$
\begin{aligned}
\dot{V}_{i j}(t)= & 2 \dot{x}^{T}(t) N_{i j} x(t)+2\left(x^{T}(t) x^{T}(t-\tau) \quad \dot{x}^{T}(t-h) \dot{x}^{T}(t)\right) M \\
& \times\left(\left(A_{i}+D_{i} K_{1 i}\right) x(t)+\left(B_{i}+D_{i} K_{2 i}\right) x(t-\tau)+C_{i} \dot{x}(t-h)-\dot{x}(t)\right) \\
= & \bar{\varsigma}^{T}(t) \bar{\Gamma}_{i j} \bar{\varsigma}(t),
\end{aligned}
$$

where

$$
\begin{aligned}
& \bar{\varsigma}^{T}(t)=\left(\begin{array}{llll}
x^{T}(t) & x^{T}(t-\tau) & \dot{x}^{T}(t-h) & \dot{x}^{T}(t)
\end{array}\right) \\
& \bar{\Gamma}_{i j}=\left[\begin{array}{cccc}
\bar{\Gamma}_{i j}^{11} & \bar{\Gamma}_{i j}^{12} & \left(A_{i}+D_{i} K_{1 i}\right)^{T} M+M C_{i} & \left(A_{i}+D_{i} K_{1 i}\right)^{T} M-M+N_{i j}^{T} \\
* & \bar{\Gamma}_{i j}^{22} & \left(B_{i}+D_{i} K_{2 i}\right)^{T} M+M C_{i} & \left(B_{i}+D_{i} K_{2 i}\right)^{T} M-M \\
* & * & M C_{i}+C_{i}^{T} M & -M+C_{i}^{T} M \\
* & * & * & -2 M
\end{array}\right], \\
& \bar{\Gamma}_{i j}^{11}=M\left(A_{i}+D_{i} K_{1 i}\right)+\left(A_{i}+D_{i} K_{1 i}\right)^{T} M, \\
& \bar{\Gamma}_{i j}^{12}=M\left(B_{i}+D_{i} K_{2 i}\right)+\left(A_{i}+D_{i} K_{1 i}\right)^{T} M, \\
& \bar{\Gamma}_{i j}^{22}=M\left(B_{i}+D_{i} K_{2 i}\right)+\left(B_{i}+D_{i} K_{2 i}\right)^{T} M .
\end{aligned}
$$

Multiplying both sides of $\bar{\Gamma}_{i j}<0$ by $\operatorname{diag}\{\bar{M}, \cdots, \bar{M}\}$. We know that (15) is equivalent to $\bar{\Gamma}_{i j}<0$, which implies $\dot{V}_{i j}(t)<0$. According to the switching law (13), at each switching instant, we have

$$
\begin{aligned}
V_{i_{k+1}}\left(t_{k+1}\right)-V_{i_{k}}\left(t_{k+1}\right) & =x^{T}\left(t_{k+1}\right)\left(P_{i_{k+1}}-P_{i_{k}}\right) x\left(t_{k+1}\right) \\
& =x^{T}\left(t_{k+1}\right) N_{i_{k+1} i_{k}} x\left(t_{k+1}\right) .
\end{aligned}
$$


From (16) and (25), we have

$$
\begin{aligned}
& V_{i_{k+1}}\left(t_{k+1}\right)-V_{i_{k}}\left(t_{k+1}\right)+V_{i_{k+2}}\left(t_{k+2}\right)-V_{i_{k+1}}\left(t_{k+2}\right) \\
& \quad=x^{T}\left(t_{k+1}\right)\left(P_{i_{k+1}}-P_{i_{k}}\right) x\left(t_{k+1}\right)+x^{T}\left(t_{k+2}\right)\left(P_{i_{k+2}}-P_{i_{k+1}}\right) x\left(t_{k+2}\right) \\
& \quad=x^{T}\left(t_{k+1}\right) N_{i_{k} i_{k+1}} x\left(t_{k+1}\right)+x^{T}\left(t_{k+2}\right) N_{i_{k+1} i_{k+2}} x\left(t_{k+2}\right) \\
& \quad \leq x^{T}\left(t_{k+1}\right) N_{i_{k} i_{k+1}} x\left(t_{k+1}\right)+x^{T}\left(t_{k+1}\right) N_{i_{k+1} i_{k+2}} x\left(t_{k+1}\right) \\
& \quad \leq x^{T}\left(t_{k+1}\right) N_{i_{k} i_{k+2}} x\left(t_{k+1}\right) .
\end{aligned}
$$

Thus,

$$
\begin{aligned}
& \sum_{p=0}^{k}\left(V_{i_{p+1}}\left(x\left(t_{p+1}\right)\right)-V_{i_{p}}\left(x\left(t_{p+1}\right)\right)\right) \\
& \leq x^{T}\left(t_{1}\right) N_{i_{0} i_{k+1}} x\left(t_{1}\right) \leq x_{0}^{T} N_{i_{0} i_{k+1}} x_{0} .
\end{aligned}
$$

Choose a class $\mathcal{G K}$ function $\beta\left(\left\|x_{0}\right\|\right)=\max _{\|x\| \leq\left\|x_{0}\right\|}\left\{\left|x^{T} N_{i j} x\right|, 1 \leq i, j \leq m\right.$. Then $x_{0}^{T} N_{i_{0} i_{k+1}} x_{0} \leq \beta\left(\left\|x_{0}\right\|\right)$ follows. The condition (26) guarantees that the overall increment of the Lyapunov-Krasovskii functional $V_{\sigma}(t)$ at switching points is decreasing. With the condition (14), asymptotic stability of the system (3) follows.

Remark 3 In order to simplify the tedious computation and highlight the design of the switching law (13), we only consider constant delays in this paper. In fact, our proposed method can be extended to deal with the case of the considered systems with time-varying delays by choosing different LyapunovKrasovskii functional (see [13] for reference).

Remark 4 If $x^{T}\left(t_{k+1}\right) N_{i_{k+1} i_{k}} x\left(t_{k+1}\right) \leq 0$, then we know from (25) that $V_{i_{k+1}}\left(t_{k+1}\right) \leq V_{i_{k}}\left(t_{k+1}\right)$ holds naturally at switching instant $t_{k+1}$. Thus conditions (15) and (16) hold naturally and condition (14) guarantees asymptotic stability of the system (3).

\section{Simulation Example}

A numerical example is given to illustrate the effectiveness of the proposed method in this section. Consider the switched neutral system (1) with two subsystems and the coefficient matrices are given by

$$
\begin{aligned}
& A_{1}=\left[\begin{array}{cc}
-5 & -0.5 \\
0 & 1
\end{array}\right], B_{1}=\left[\begin{array}{cc}
-0.4 & 0 \\
0 & -0.6
\end{array}\right], C_{1}=\left[\begin{array}{cc}
-0.1 & 0.5 \\
0.1 & 0
\end{array}\right], D_{1}=\left[\begin{array}{l}
1 \\
0
\end{array}\right] \\
& A_{2}=\left[\begin{array}{cc}
1 & 0 \\
-1 & -5
\end{array}\right], B_{2}=\left[\begin{array}{cc}
-0.4 & 0 \\
-1 & -0.3
\end{array}\right], C_{2}=\left[\begin{array}{cc}
-0.1 & 0.5 \\
0.1 & 0
\end{array}\right], D_{2}=\left[\begin{array}{l}
0 \\
1
\end{array}\right]
\end{aligned}
$$

Suppose $\tau=h=0.1$. Choose the parameters $\alpha_{12}=\alpha_{21}=-10$. By solving the conditions in Theorem 1, we obtain

$$
\begin{gathered}
P_{1}=\left[\begin{array}{cc}
3.1026 & -0.2902 \\
-0.2902 & 7.1135
\end{array}\right], P_{2}=\left[\begin{array}{cc}
4.1236 & -0.2940 \\
-0.2940 & 5.6144
\end{array}\right], \\
N_{12}=\left[\begin{array}{cc}
-0.3919 & 0.0741 \\
0.0741 & -1.0959
\end{array}\right], N_{21}=\left[\begin{array}{cc}
-0.4436 & 0.0752 \\
0.0752 & -1.1319
\end{array}\right]
\end{gathered}
$$




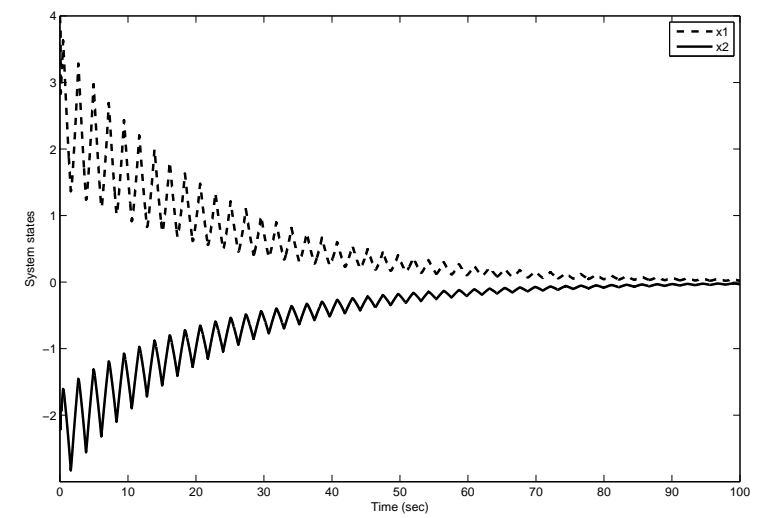

Fig. 2 The state trajectories of the system under the switching law (29).

and

$$
\begin{aligned}
& u_{1}(t)=\left(\begin{array}{ll}
4.4139 & 0.6726
\end{array}\right) x(t)+\left(\begin{array}{ll}
0.4001 & -0.0365
\end{array}\right) x(t-0.1), \\
& u_{2}(t)=\left(\begin{array}{ll}
1.1032 & 4.5679
\end{array}\right) x(t)+\left(\begin{array}{ll}
0.9853 & 0.3001
\end{array}\right) x(t-0.1) .
\end{aligned}
$$

According to (13), the switching law is given by

$$
\sigma(t)=\left\{\begin{array}{cl}
1, & \text { if } x(0) \in \Omega_{1} \text { or }\left(x(t) \in \Omega_{1} \text { and } \sigma\left(t^{-}\right)=1\right) \\
& \text { or }\left(x(t) \in \Omega_{21} \text { and } \sigma\left(t^{-}\right)=2\right) \\
2, & \text { if } x(0) \in \Omega_{2} \text { or }\left(x(t) \in \Omega_{2} \text { and } \sigma\left(t^{-}\right)=2\right) \\
\text { or }\left(x(t) \in \Omega_{12} \text { and } \sigma\left(t^{-}\right)=1\right)
\end{array}\right.
$$

where

$$
\begin{aligned}
& \Omega_{1}=\left\{x(t) \in \mathbb{R}^{2} \mid x^{T}(t)\left[\begin{array}{cc}
-1.4130 & 0.0778 \\
0.0778 & 0.4032
\end{array}\right] x(t) \leq 0\right\}, \\
& \Omega_{2}=\left\{x(t) \in \mathbb{R}^{2} \mid x^{T}(t)\left[\begin{array}{cc}
0.5775 & 0.0714 \\
0.0714 & -2.6310
\end{array}\right] x(t) \leq 0\right\},
\end{aligned}
$$

$\Omega_{12}$ and $\Omega_{21}$ are the boundaries of $\Omega_{1}$ and $\Omega_{2}$, respectively.

The state trajectories of the closed-loop system with $x_{0}=(4-2)$ are depicted in Fig.2. From Fig.2, we can see that the state is convergent. The control input and the switching signal are shown in Fig.3 and Fig.4, respectively.

In order to show the advantage of our proposed method, we let $N_{12}=$ $N_{21}=0$. Then, the conditions (15) and (16) in Theorem 1 hold naturally. By solving the condition (14), we obtain

$$
P_{1}=\left[\begin{array}{cc}
2.1168 & -0.1527 \\
-0.1527 & 4.7894
\end{array}\right], P_{2}=\left[\begin{array}{cc}
2.8481 & -0.1560 \\
-0.1560 & 3.7455
\end{array}\right]
$$

and

$$
u_{1}(t)=\left(\begin{array}{ll}
4.1022 & 0.6409
\end{array}\right) x(t)+(0.4001-0.0267) x(t-0.1)
$$




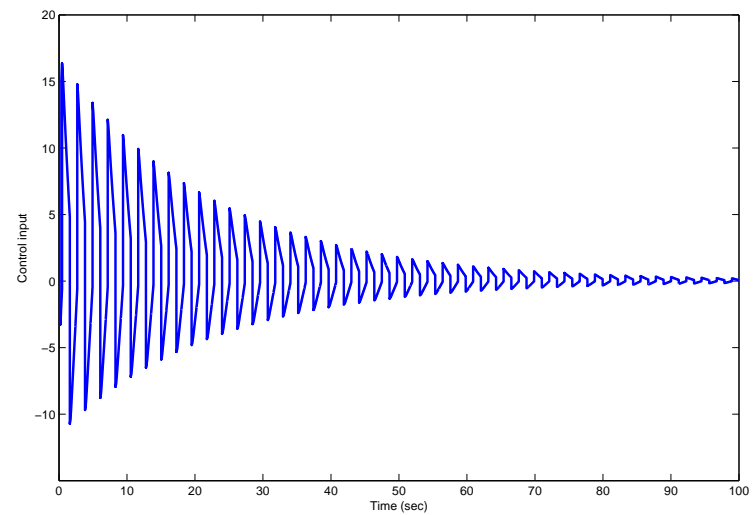

Fig. 3 Control input under the switching law (29).

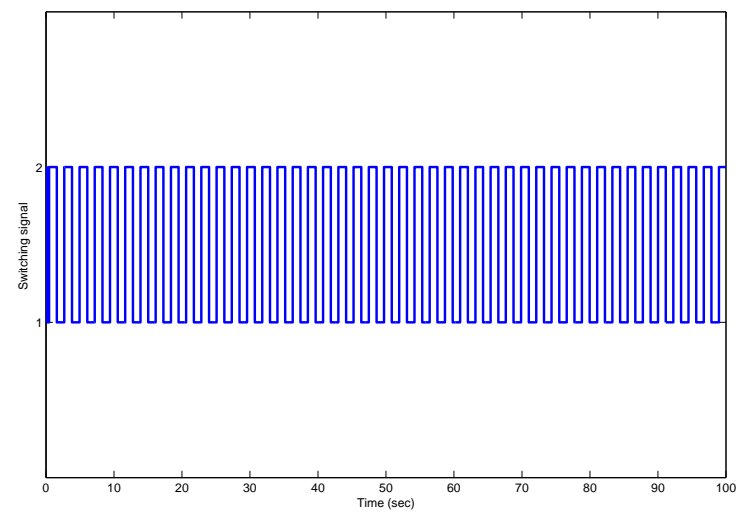

Fig. 4 The switching signal (29).

$$
u_{2}(t)=\left(\begin{array}{ll}
1.0879 & 4.4937
\end{array}\right) x(t)+(0.99320 .3001) x(t-0.1) .
$$

The switching law (13) degenerates into the min-switching strategy:

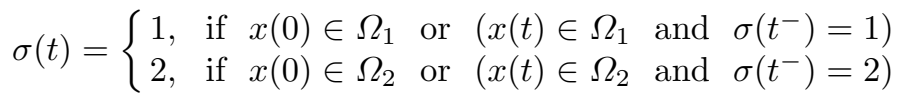

where

$$
\begin{gathered}
\Omega_{1}=\left\{x(t) \in \mathbb{R}^{2} \mid x^{T}(t)\left[\begin{array}{cr}
-0.7313 & 0.0032 \\
0.0032 & 1.0440
\end{array}\right] x(t) \leq 0\right\}, \\
\Omega_{2}=\left\{x(t) \in \mathbb{R}^{2} \mid x^{T}(t)\left[\begin{array}{cr}
0.7313 & -0.0032 \\
-0.0032 & -1.0440
\end{array}\right] x(t) \leq 0\right\} .
\end{gathered}
$$

The state trajectories of the closed-loop system are depicted in Fig.5. From Fig.5, we find that asymptotic stability of the closed-loop system can still be 


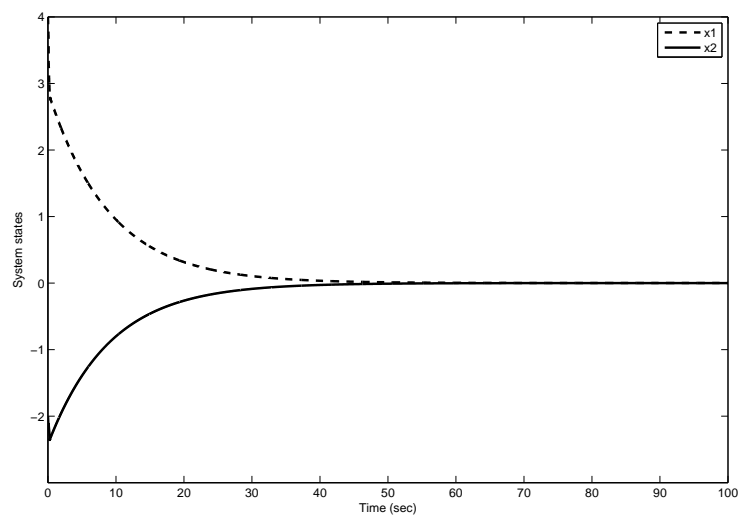

Fig. 5 The state trajectories of the system under the switching law (32).

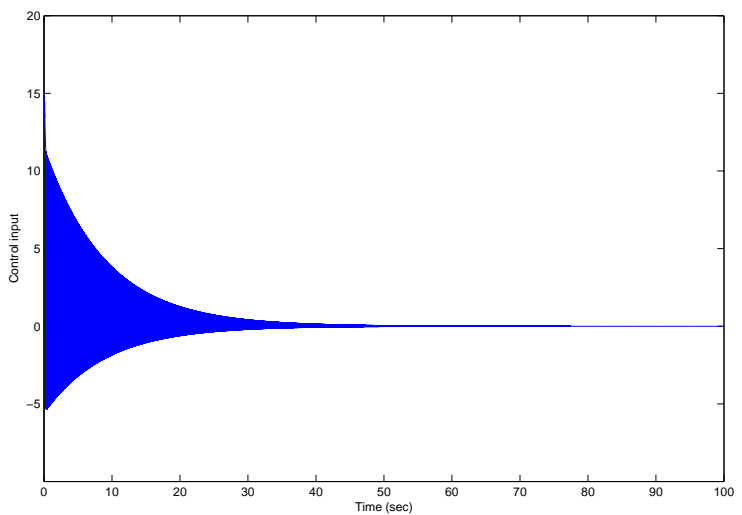

Fig. 6 Control input under the switching law (32).

guaranteed. However, from Fig.6-Fig.7, we see that the min-switching strategy without hysteresis leads to infinitely fast switchings, which is undesirable in practice. However, our proposed switching strategy can avoid this behavior.

\section{Conclusion}

We have presented a stabilization result for a class of continuous-time switched linear neutral systems by co-designing a hysteresis-based switching strategy and a set of candidate controllers. We also have obtained a delay-dependent stability criterion by finding a multiple generalized Lyapunov-Krasovskii functional. Future work will focus on the discrete-time case of the studied problem in this paper and further apply it to the sensor networks [36]. 


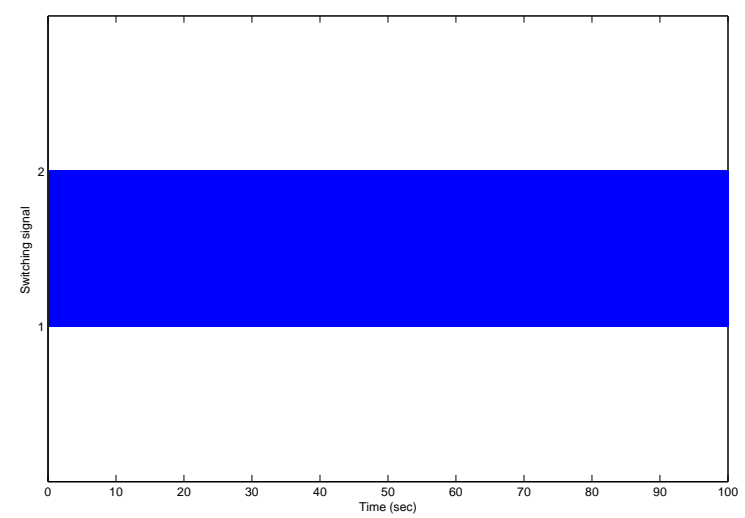

Fig. 7 The switching signal (32).

Acknowledgements This work was supported by the National Nature Science Foundation of China under grants 61503041 and 61473063.

\section{References}

1. Barkhordari, Y., Jahed-Motlagh, M.: Stabilization of a CSTR with two arbitrarily switching modes using model state feedback linearization. Chem. Eng. J., 155(3), 838-843(2009)

2. Branicky, M. S.: Multiple Lyapunov functions and other analysis tools for switched and hybrid systems. IEEE T. Automat. Contr., 43(4), 475-482 (1998)

3. Chen, W., Zheng, W.: Delay-dependent robust stabilization for uncertian neutral systems with distributed delays. Automatica, 43(1), 95-104(2007)

4. Daafouz, J., Riedinger, P., Iung, C.: Stability analysis and control synthesis for switched systems: a switched Lyapunov function approach. IEEE T. Automat. Contr., 47(11), 1883$1887(2002)$

5. Fu, J., Ma, R., Chai, T.: Global finite-time stabilization of a class of switched nonlinear systems with the powers of positive odd rational numbers. Automatica, 54, 360-373(2015)

6. Gu, K., Kharitonov, V., Chen, J.: Stability of time-delay systems. Birkhauser, (2003)

7. Hale, J., Lunel, Verduyn S.: Introduction to functional differential equations. SpringerVerlag, New York (1993)

8. He, Y., Wu, M., She, J., Liu, G.: Delay-dependent robust stability criteria for uncertain neutral systems with mixed delays. Syst. Control Lett., 51(1), 57-65(2004)

9. Kolmanovskii, V., Nosov, V.: Stability of functional differential equations. Academic, London (1986)

10. Krishnasamy, R., Balasubramaniam, P.: A descriptor system approach to the delaydependent exponential stability analysis for switched neutral systems with nonlinear perturbations. Nonlinear Analysis: Hybrid Systems, 15, 23-36(2015)

11. Kuang, Y.: Delay differential equations with application in population dynamics. New York: Academic Press, 191(1993)

12. Li, T., Zhao, J., Dimirovski, M.: Stability and $L_{2}$-gain analysis for switched neutral systems with mixed time-varying delays. J. Frankl. Inst., 348(9), 2237-2256(2011)

13. Li, T., Dimirovski, G., Liu, Y., Zhao, J.: Improved stability of a class of switched neutral systems via Lyapunov-Krasovskii functional and an average dwell-time scheme. Int. J. Syst. Sci., 44(6), 1076-1088 (2013)

14. Li, T., Zhao, J., Qi, Y.: Switching design of stabilizing switched neutral systems with application to lossless transmission lines. IET Control Theory A., 17(8), 2082-2091(2014) 
15. Li, T., Zhao, J., Dimirovski, G.: Hysteresis switching design for stabilization of a class of switched neutral systems. Asian J. Control, 15(4), 1149-1157 (2013)

16. Liberzon, D.: Switching in systems and control. Boston: Birkhauser (2003)

17. Lin, H., Antsaklis, P. J.: Stability and stabilizability of switched linear systems: a survey of recent results. IEEE T. Automat. Contr., 54, 308-322(2009)

18. Lin, J., Fei, S., Gao, Z.: Stabilization of discrete-time switched singular time-delay systems under asynchronous switching. J. Frankl. Inst., 349(5), 1808-1827(2012)

19. Liu, D., Zhong, S., Liu, X., Huang, Y.: Stability analysis for uncertain switched neutral systems with discrete time-varying delay: a delay-dependent method. Math. Comput. Simulat., 80(2), 436-448(2009)

20. Lu, B., Wu, F.: Switching LPV control designs using multiple parameter-dependent Lyapunov functions. Automatica, 40(11), 1973-1980(2004)

21. Ma, D., Zhao, J.: Stabilization of networked switched linear systems: an asynchronous delay system approach. Syst. Control Lett., 77, 46-54(2015)

22. Ma, R., Zhao, J.: Backstepping design for global stabilization of switched nonlinear systems in lower triangular form under arbitrary switchings. Automatica, 46(11), 18191823(2010)

23. Niu, B., Zhao, J.: Barrier Lyapunov functions for the output tracking control of constrained nonlinear switched systems. Syst. Control Lett., 62(10), 963-971(2013)

24. Richard, J.: Time-delay systems: an overview of some recent advances and open problems. Automatica, 39, 1667-1694(2003)

25. Saldivar, B., Mondie, S., Loiseau, J. J., Rasvan,V.: Exponential stability analysis of the drilling system described by a switched neutral type delay equation with nonlinear perturbations. IEEE Conf. Decision Control and European Control Conference, Orlando, USA, 4164-4169(2011)

26. Sun, X., Wu, D., Liu, G., Wang, W.: Input-to-state stability for networked predictive control with random delays in both feedback and forward channels. IEEE T. Ind. Electron., 61(7), 3519-3526(2014)

27. Sun, X., Fu, J., Sun, H., Zhao, J.: Stability of linear switched neutral delay systems. Proceedings of the CSEE, 25(23), 42-46(2005)

28. Wang, S., Shi, T., Zhang, L., Jasra, A., Zeng, M.: Extended finite-time $H_{\infty}$ control for uncertain switched linear neutral systems with time-varying delays. Neurocomputing, 152(25), 377-387(2015)

29. Wang, Y., Zhao, J., Jiang, B.: Stabilization of a class of switched linear neutral systems under asynchronous switching. IEEE T. Automat. Contr., 58(8), 2114-2119(2013)

30. Wang, Y., Sun, X., Zhao, J.: Stabilization of a class of switched stochastic systems with time delays under asynchronous switching. Circ. Syst. Signal Pr., 32(1), 347-360(2013)

31. Xiang, Z., Sun, Y., Chen, Q.: Robust reliable stabilization of uncertain switched neutral systems with delayed switching. Appl. Math. Comput., 217(23), 9835-9844(2011)

32. Xiang, Z., Sun, Y., Mahmoud, M.: Robust finite-time $H_{\infty}$ control for a class of uncertain switched neutral systems. Commun. Nonlinear Sci., 17(4), 1766-1778(2012)

33. Xiong, L., Zhong, S., Ye, M., Wu, S.: New stability and stabilization for switched neutral control systems. Chaos Soliton. Fract., 42(3), 1800-1811(2009)

34. Zhang, D., Yu, L., Wang, Q., Ong, C. J.: Estimator design for discrete-time switched neural networks with asynchronous switching and time-varying delay, IEEE T. Neural Networks and Learning Systems, 23(5), 827-834(2012)

35. Zhang, D., Yu, L.: Exponential stability analysis for neutral switched systems with interval time-varying mixed delays and nonlinear perturbations. Nonlinear Analysis: Hybrid Systems, 6(2), 775-786(2012)

36. Zhang, D., Cai, W. J., Xie, L. H., Wang, Q. G.: Nonfragile distributed filtering for T-S fuzzy systems in sensor networks, IEEE T. Fuzzy Syst., 23(5), 1883-1890(2015)

37. Zhang, L., Gao, H.: Asynchronously switched control of switched linear systems with average dwell time. Automatica, 46, 953-958(2010)

38. Zhang, Y., Liu, X., Zhu, H., Zhong, S.: Stability analysis and control synthesis for a class of switched neutral systems. Appl. Math. Comput., 190(2), 1258-1266(2007)

39. Zhao, J., Hill, D.: On stability, $L_{2}$-gain and $H_{\infty}$ control for switched systems. Automatica, 44(5), 1220-1232(2008) 
40. Zhao, X., Zhang, L., Shi, P., Liu, M.: Stability and stabilization of switched linear systems with mode-dependent average dwell time. IEEE T. Automat. Contr., 57(7), 1809$1815(2012)$

41. Zhao, X., Yin, Y., Yang, H., Li, R.: Adaptive control for a class of switched linear systms using state-dependent switching. Circ. Syst. Signal Pr., 34(11), 3681-3695 (2015)

42. Zhu, X., Yang, G.: Delay-dependent stability criteria for systems with differentiable time delays. Acta Automatica Sinica, 34(7), 756-771(2008) 